\title{
La estructura de la conducta prosocial. Su aproximación mediante el modelo bifactorial de la Teoría de la Respuesta al Ítem Multidimensional
}

\author{
The structure of prosocial behavior. Its approach through the Multidimensional Item \\ Response Theory's two-factor model
}

\author{
Sofía Esmeralda Aunéa,b,*, Facundo Juan Pablo Abal ${ }^{\mathrm{a}, \mathrm{b}}$, Horacio Félix Attorresia \\ anstituto de Investigaciones, Facultad de Psicología, Universidad de Buenos Aires, Argentina \\ ${ }^{b}$ Consejo Nacional de Investigaciones Científicas y Técnicas (CONICET), Argentina
}

\section{Resumen}

Problema y objetivos: conocer si la estructura de la conducta prosocial medida por la Escala de Conducta Prosocial (ECP) puede ser representada por un factor general, la prosocialidad, combinado con los factores específicos, las subescalas de la ECP. Método: se trató de una muestra por accesibilidad de 692 participantes (65\% mujeres), residentes en Gran Buenos Aires e interior de la Argentina. Se comprobaron los supuestos de unidimensionalidad y dependencia local, posteriormente se comparó el ajuste relativo entre tres modelos de la Teoría de la Respuesta al Ítem: Modelo de Respuesta Graduada (MRG), Modelo de Respuesta Graduada Multidimensional (MRGM) y Modelo de Respuesta Graduada Bifactorial (MRGB). Resultados: el MRGB fue el modelo con mejor ajuste relativo, se implementó dicho modelo calculándose los parámetros condicionales y marginales. Asimismo, se calculó el índice ECV. Conclusiones: los ítems de la ECP fueron influenciados primariamente por la prosocialidad. La subescala Ayuda explicó una proporción importante de la varianza común. En cambio, la subescala Confortar, considerada como factor específico, explicó una parte pequeña de dicha varianza.

Palabras clave: conducta prosocial, prosocialidad, teoría de la respuesta al ítem multidimensional, modelo bifactorial.

\section{Para citar este artículo:}

Aune, S. E., Abal, F. J. P., \& Attorresi, H. F. (2019). La estructura de la conducta prosocial. Su aproximación mediante el modelo bifactorial de la Teoría de la Respuesta al Ítem Multidimensional. Liberabit, 25(1), 4156. doi: https://doi.org/10.24265/liberabit.2019.v25n1.04

\begin{abstract}
Problem and objectives: to find out if the structure of the prosocial behavior measured by the Prosocial Behavior Scale (PBS) can be represented by a general factor, i.e. prosociality, combined with specific factors: the subscales of the PBS. Method: the sample consisted of 692 subjects (65\% females) recruited by a convenience sampling method and living in Greater Buenos Aires and inland areas of Argentina. The assumptions of unidimensionality and local dependence were checked. Then the relative fit was compared between three models of the Item Response Theory: Graded Response Model (GRM), Multidimensional Graded Response Model (MGRM) and Two-Factor Graded Response Model (TGRM). Results: the TGRM showed the best relative fit. This model was implemented by calculating the conditional and marginal parameters. Likewise, the ECV index was calculated. Conclusions: the PBS items were primarily influenced by prosociality. The Help subscale explained a significant proportion of the common variance. In contrast, the Comfort subscale, considered as a specific factor, explained a small part of said variance.
\end{abstract}

Keywords: prosocial behavior, prosociality, multidimensional item response theory, two-factor model.

Este es un artículo Open Access bajo la licencia Creative Commons Atribución-NoComercial-CompartirIgual 4.0

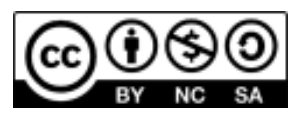

Universidad de San Martín de Porres, Lima - Perú 41 http://ojs3.revistaliberabit.com 


\section{Introducción}

Se consideran como prosociales a las acciones voluntarias realizadas para beneficiar a otros (Dunfield, 2014; Eisenberg, Eggum, \& Giunta, 2010). Previamente a realizar un comportamiento prosocial, se observan e interpretan las necesidades ajenas y se ponderan con la propia capacidad percibida para darles alivio (Caprara, Alessandri, \& Eisenberg, 2012). La conducta prosocial como constructo surge históricamente como alternativa al altruismo (Auné, Blum, Abal, Lozzia \& Attorresi, 2014). Este último generaba problemas teóricos y metodológicos en la determinación de que una conducta sea realmente altruista. La conducta prosocial, en cambio, no está asociada a una motivación específica.

El constructo conducta prosocial ha sido concebido clásicamente como multidimensional. Las primeras teorías proponen una enorme cantidad de subdimensiones. Este es el caso de Roche (1998) que distingue entre: Ayuda física, Servicio físico, Dar, Ayuda verbal, Consuelo verbal, Confirmación y valorización positiva del otro, Escucha profunda, Empatía, Solidaridad y presencia positiva, y Unidad.

Posteriormente, tal vez debido a la aplicación de análisis factoriales exploratorios y/o confirmatorios, se arriba a estructuras más simples y parsimoniosas. Es decir, mientras que las primeras teorías acerca de la conducta prosocial se basaban en distinciones minuciosas y detalladas tipologías del constructo, las conceptualizaciones más recientes realizan diferenciaciones más simples y globales. Esto se puede corresponder con la búsqueda de coherencia entre la operacionalización del constructo mediante escalas de evaluación psicológica y la teoría acerca del mismo.

En 2002, Carlo y Randall proponen seis dimensiones para el constructo: Comportamiento altruista, Comportamiento reactivo, Comportamiento emocional, Comportamiento público, Comportamiento anónimo y Comportamiento en situaciones de urgencia. En 2007, Hay y Cook formulan una división de las conductas prosociales entre Sentimientos por el otro, Trabajar con otro y Atender a otro; en tanto que Warneken y Tomasello (2009) aíslan las subdimensiones Confortar, Compartir, Informar y Ayuda instrumental. Dunfield (2014) distingue entre Ayudar, Compartir y Confortar. Más recientemente Auné y Attorresi (2017) sostienen una estructura de dos dimensiones: Confortar y Ayuda.

Particularmente, Caprara et al. (2012) proponen la existencia de una tendencia subyacente de la personalidad a realizar acciones prosociales en diferentes momentos y situaciones, la cual consideran que conforma una única dimensión. Conceptualizaciones coherentes con esta perspectiva ya habían sido sostenidas por otros autores como Penner, Fritzsche, Craiger y Freifeld (1995). Ellos utilizan el término de personalidad prosocial para referirse a la tendencia continua a preocuparse por el bienestar y los derechos de los demás realizando acciones al respecto.

La prosocialidad ha sido ampliamente investigada en la adolescencia y niñez (e.g., Carrasco \& Trianes, 2010). También la idea de la prosocialidad como aspecto de la personalidad se ha utilizado como constructo en la etapa adulta. En esta línea, se ha estudiado la relación de la prosocialidad con la tendencia a realizar acciones proambientales (Cuadrado, Tabernero, García, Luque, \& Seibert, 2017), con rasgos psicopáticos de la personalidad (Gatner, Douglas, \& Hart, 2016) y con la vivencia de eventos traumáticos (Strakatý, 2016).

\section{Medición de la conducta prosocial}

Dada la importancia conceptual y aplicada de la conducta prosocial en diversos ámbitos de la Psicología tales como la Psicología Clínica, Social y Ambiental, se han desarrollado distintas escalas para medirla. Es necesario destacar que la creación y adaptación de instrumentos para su evaluación se ha realizado inicialmente a fines de los 80 y en la década de los 90. Esos instrumentos se orientan en su enorme mayoría a niños y adolescentes. Se ha arribado a esta 
conclusión luego del relevo minucioso de escalas para la medición de la conducta prosocial realizado en Auné et al. (2014). Caprara, Steca, Zelli y Capanna (2005) sitúan que el estudio de la prosocialidad ha sido abordado primordialmente por la psicología del desarrollo, existiendo un menor conocimiento de su relación con el bienestar personal y ajuste social en etapas posteriores. Algunos de los instrumentos clásicos de evaluación son, por ejemplo, el Inventario de Habilidades Sociales para Adolescentes (Teenage Inventory of Social Skills [TISS]) de Inderbitzen y Foster (1992) que mide la conducta prosocial y agresiva de los adolescentes en las relaciones con sus pares. Otro test muy relevante es la Escala de Conducta Prosocial (Prosocial Behavior Scale [PBS]) de Caprara y Pastorelli (1993), en este caso para ser aplicado en niños. Una excepción es la Batería de Personalidad Prosocial (Prosocial Personality Battery [PSB]) de Penner et al. (1995) que mide la conducta prosocial en adultos.

La construcción de instrumentos de evaluación de la conducta prosocial más breves y cuyos destinatarios sean adultos cobra fuerza en el presente siglo. En 2005 se publica la Escala para la Medición de la Prosocialidad en Adultos (Prosocialness Scale for Adults [PSA]) de Caprara et al. (2005) que consta de 16 ítems. Los autores examinan la PSA mediante la Teoría de la Repuesta al Ítem (TRI), hallando que incluso los sujetos con bajo nivel de prosocialidad tendían a responder favorablemente a los ítems.

\section{La Escala de Conducta Prosocial (ECP)}

En 2017 se presentó un nuevo instrumento: la Escala de Conducta Prosocial (ECP) de Auné y Attorresi. En este estudio se aplicó sobre la ECP un análisis factorial exploratorio donde el método de extracción de factores fue el de mínimos cuadrados no ponderados (Unweighted Least Squares [ULS]) con rotación promax sobre matrices policóricas. Se arribó a una estructura de dos factores ya que la unidimensionalidad, la estructura más simple, no pudo sostenerse.
En referencia a la base conceptual, se definió a la conducta prosocial como un fenómeno complejo que involucra acciones de los individuos basadas en creencias y sentimientos, y que describe la forma en que estos se orientan hacia los otros al realizar conductas solidarias. El desarrollo de la ECP, asimismo, fue coherente con la definición de González (2000) que entiende por conducta prosocial a toda conducta social positiva con o sin motivación altruista.

Al seleccionarlos, se buscó que los ítems evalúen diferentes grados de beneficio hacia el otro y distintos beneficiarios de la acción prosocial. Se procuró que tuvieran claridad, comprensibilidad, relevancia y representatividad del constructo. Posteriormente, el pool de ítems fue depurado sucesivamente con análisis factoriales.

Sus 15 ítems se dividen en dos subescalas: Confortar y Ayuda. Confortar (7 ítems) está compuesta por comportamientos que manifiestan empatía, comprensión, refuerzo y soporte emocional. La subescala Ayuda (8 ítems) describe comportamientos de asistencia, cuidado y compromiso con los otros. Se incluyeron reactivos que, por su contenido, presumiblemente puedan medir en niveles moderados o altos de conducta prosocial. En relación con la consistencia interna, el alfa de Cronbach de la subescala Confortar fue de .77 y de la subescala Ayuda, .85. En conjunto, Confortar y Ayuda explicaron el $50.61 \%$ de la varianza, correlacionando moderadamente en .49. Las cargas factoriales que expresan la importancia relativa del factor para explicar el puntaje individual de cada ítem y las correlaciones del ítem con el factor que le corresponde fueron elevadas, oscilando entre .40 y .89 (Auné \& Attorresi, 2017).

Se han hallado reiteradamente asociaciones positivas, de moderadas a altas, entre las diferentes dimensiones de la conducta prosocial (e.g., Penner et. al, 1995). Asimismo, los puntajes de distintos instrumentos de medición de la conducta prosocial han correlacionado positivamente entre sí (e.g., Carlo \& 
Randall, 2002; Ladd \& Profilet, 1996). Estos resultados repetidos podrían estar sugiriendo que las dimensiones de la conducta prosocial son constructos diferentes, pero, al mismo tiempo, superpuestos en cierto grado. Dicho patrón es coherente con la idea de que representan distintas dimensiones de un constructo latente subyacente: la tendencia a realizar acciones prosociales, también denominada prosocialidad.

Sería de utilidad y de importancia teórica relevar la existencia de la prosocialidad, así como también, su relación con las diferentes dimensiones que se han encontrado para explicar la conducta prosocial. Para explorar empíricamente dichas relaciones, se utilizará el instrumento de más reciente publicación para la medición de la conducta prosocial, la ECP de Auné y Attorresi (2017). La misma podría estar midiendo, simultáneamente, un factor general subyacente, la prosocialidad; y al mismo tiempo mediante sus dos subescalas, factores más específicos. En dicho caso, cada uno de los ítems de la ECP podría representar al factor general de la prosocialidad y a su vez a la subescala de pertenencia, Confortar o Ayuda, en algún grado. Un modelo de estructura bifactorial sería tal vez adecuado para representar la multidimensionalidad relevante para un conocimiento más profundo del constructo (Reise, Moore, \& Haviland, 2010).

Los modelos bifactoriales asumen que las respuestas a los ítems pueden ser influenciadas por múltiples variables latentes. Más específicamente, por un factor general (FG) y una serie de factores específicos (FE) (Cai, 2015; Toland, Sulis, Giambona, Porcu, \& Campbell, 2017). La varianza común de la totalidad de los ítems es explicada por el FG, es por esto que el modelo considera que el FG y los FE no están correlacionados, es decir que son ortogonales entre sí (Gibbons \& Hedeker, 1992). Si bien los modelos bifactoriales se pueden implementar desde la teoría clásica de los test, es solo mediante la TRI donde se obtienen modelos bifactoriales con información completa (Bock \& Gibbons, 2010). El análisis factorial basado en la TRI se considera completo porque utiliza la totalidad de la información proveniente de las respuestas categóricas originales y no depende de índices de asociación tales como coeficientes de correlación policóricos o tetracóricos.

El objetivo principal de la presente investigación fue, entonces, conocer si la estructura de la conducta prosocial medida por la ECP puede ser representada por un FG combinado con los FE. La prosocialidad como tendencia subyacente de la personalidad estaría representada por el FG, mientras cada una de las subescalas sería un FE. Se hipotetizó, por consiguiente, que una estructura bifactorial daría cuenta de la covariación entre los ítems de la ECP.

\section{Método}

\section{Participantes}

Se trató de una muestra por accesibilidad de 692 participantes (65\% mujeres, media $=27.1$ años, $D E=$ 6.26) residentes en Gran Buenos Aires (25\%) e interior de la Argentina (75\%). Respecto del interior de la Argentina, 31\% se ubicaba en la provincia de Córdoba, $18 \%$ en la provincia de Santa Fe, $16 \%$ en el interior de la provincia de Buenos Aires, 9\% en la provincia de Salta, mientras que el resto se repartía en otras provincias argentinas. El 13\% contaba con un nivel educativo de como máximo secundaria incompleta (12.4\% de las mujeres y 16.3\% de los varones), el 24\% había finalizado estudios secundarios (23.9\% de las mujeres y 22.6\% de los varones), mientras que el 63\% restante había, como mínimo, comenzado estudios terciarios (63.7\% de las mujeres y $61.1 \%$ de los varones). Para las mujeres la media de edad fue de $27.26(D E=6.25)$, mientras que para los varones su media fue de $26.52(D E=6.12)$. Se utilizó la misma muestra que en Auné y Attorresi (2017).

\section{Instrumentos}

Cuestionario sociodemográfico. Se trata de un cuestionario de tipo encuesta que reveló las siguientes variables: género, edad, lugar de residencia y nivel de estudios. 
Escala de Conducta Prosocial (Auné \& Attorresi, 2017). Los ítems que la componen son los siguientes: 1) Relego mi beneficio personal para ayudar a otros, 2) Realizo actividades como voluntario, 3) Cuando siento que alguien está mal, le demuestro que lo entiendo, 4) Actúo como bastón de los demás, 5) Siento todo dolor ajeno como propio, 6) Me comprometo con causas nobles, 7) Dedico un tiempo importante de mi vida a mejorar el mundo, 8) Me ocupo del bienestar de cualquier individuo, grupo o comunidad, 9) Me quedo con lo justo y necesario para vivir y reparto todo lo demás, 10) Me pongo en el lugar del otro, 11) Participo en actividades solidarias, 12) Hago donaciones a organizaciones benéficas, 13) $\mathrm{Si}$ una persona me cuenta un conflicto, intento que comprenda el punto de vista de la otra parte, 14) Intento «levantar» la autoestima a mis amigos y 15) Aconsejo a conocidos sobre trabajo. La modalidad de respuesta se especificó con una escala Likert de seis opciones que refleja la frecuencia con que se realiza la acción (1 = Nunca, 2 = Casi Nunca, $3=$ A Veces, $4=$ Con Frecuencia, 5 = Casi Siempre, 6 = Siempre) .

\section{Procedimiento}

La información se recogió mediante una encuesta creada en la plataforma Google Forms, compartida en redes sociales virtuales. Se solicitó el consentimiento informado y se aclaró que la participación era enteramente voluntaria y anónima, con fines de investigación y que se podía cesar en el momento deseado. El diseño de investigación propuesto sigue las normativas internacionales planteadas por la Asociación Americana de Psicología (American Psychological Association [APA], 2010). Fueron criterios de exclusión de la muestra haber omitido más del $10 \%$ de las respuestas y tener una edad menor a 18 años cumplidos.

\section{Análisis de datos \\ Comprobación de los supuestos de unidimensionalidad e independencia local}

Con el fin de explorar la presencia de datos anómalos multivariados, se calculó la distancia de Mahalanobis considerando como punto de corte $p \leq$ .001 (Tabachnick \& Fidell, 2001). De esta forma, se detectaron 25 outliers que se eliminaron de la muestra. Por lo tanto, resultó un nuevo tamaño muestral de 667 participantes sobre el cual se realizaron los subsiguientes análisis.

En primer lugar, se obtuvieron los siguientes estadísticos descriptivos univariados para cada ítem: media, desvío estándar, asimetría y curtosis. Posteriormente, se realizó la comprobación de los supuestos de los modelos tradicionales de la TRI (Ayala, 2009) sobre los 15 ítems: unidimensionalidad e independencia local. El supuesto de unidimensionalidad se examinó mediante análisis factorial exploratorio (AFE), utilizando como método de extracción de factores el de mínimos cuadrados no ponderados robustos con muestreo bootstrap. Se observó, mediante la implementación óptima del análisis paralelo (Timmerman \& Lorenzo-Seva, 2011), la cantidad sugerida de dimensiones que se complementó con los índices: error medio cuadrático de aproximación (Root Mean Square Error of Approximation [RMSEA]) y raíz media cuadrática residual (Root Mean Square Residual [RMSR]). Aunque el análisis paralelo sugirió la existencia de una dimensión preponderante, se halló una pobre adecuación a la unidimensionalidad señalada por un RMSEA de .111. Para que el modelo se considere ajustado, el RMSEA debe ser menor o igual que .08 (Hu \& Bentler, 1999). La RMSR fue de .103 mientras que el valor esperado para un modelo aceptable era de menor o igual a .039 según el criterio de Kelley. En cuanto a los residuos estandarizados, estos tuvieron un valor máximo de 8.820, ciertamente muy alto. Estos indicadores señalan que las respuestas a los 15 ítems de la ECP no presentan el grado de unidimensionalidad necesario como para considerar satisfecho dicho supuesto. 
El segundo supuesto, la independencia local, se examinó ajustando a los ítems el Modelo de Respuesta Graduada (MRG; Samejima, 1969) tradicional. Se halló que un par de ítems no cumplió este supuesto. La posible dependencia local fue señalada por un índice $\chi^{2}$ LD (Chen \& Thissen, 1997) notoriamente mayor que 10 .

Además, se estudió el Funcionamiento Diferencial del Ítem (Differential Ítem Functioning [DIF]) según el género para descartar una influencia de esta variable y así, una disminución de la validez. Este estudio se realizó con el test de Wald modificado (Cai, 2012; Langer, 2008).

\section{Comparación entre modelos de la TRI}

Dado que evidentemente no se cumplieron los supuestos para que sea adecuado aplicar a la ECP un modelo tradicional de la TRI, quedó justificada empíricamente la aplicación de un modelo bifactorial. Particularmente, se implementó el Modelo de Respuesta Graduada Bifactorial (MRGB; Gibbons et al., 2007). Para este modelo confirmatorio se especificaron tres factores: el FG y un FE para cada una las subescalas Confortar y Ayuda. Su ajuste se comparó con otros dos modelos: a) el MRG tradicional (Samejima, 1969) y b) el Modelo de Respuesta Graduada Multidimensional (MRGM, Muraki, \& Carlson, 1995). El primero se corrió para utilizarlo como base comparativa, aunque no se cumplieron los supuestos para su aplicación. Este es un procedimiento usual para dar cuenta de las ventajas de modelos menos restrictivos (e.g., Ouyang, Xin, \& Chen, 2016). El MRGM es la extensión multidimensional del MRG, tratándose de la misma función generalizada para dos o más rasgos latentes (Toland et al., 2017). Se impuso una estructura de dos factores correlacionados correspondientes con las dos subescalas de la ECP, es decir que en el MRGM no se considera la existencia del FG.

Se compararon los resultados del MRG, MRGM y MRGB a partir del índice -2 por el logaritmo de la verosimilitud (-2 log likelihood, -2LL), el Criterio de Información de Akaike (Akaike Information Criterion, AIC), el Criterio de Información Bayesiano (Bayesian Information Criterion, BIC), el grado de ajuste global, la cantidad de ítems que ajustan al modelo, la cantidad de pares de ítems con dependencia local detectados y el valor máximo del índice $\chi^{2} \mathrm{LD}$. Cuanto más pequeños sean -2LL, AIC y BIC, esto señala mejor ajuste relativo. El ajuste global se obtuvo con el estadístico $M_{2}$ (Maydeu-Olivares \& Joe, 2005, 2006) y su RMSEA asociado. El modelo ajusta globalmente si RMSEA es menor que .05. Comparativamente, menores valores del estadístico $M_{2}$ y de RMSEA indican un mejor ajuste relativo. La cantidad de ítems que ajustaron a cada modelo se calculó con el índice S- $\chi^{2}$ (Bjorner et al., 2007; Orlando \& Thissen, 2000, 2003) utilizando el procedimiento de ajuste de Benjamini-Hochberg (1995) sobre el nivel de significación. Por último, el $\chi^{2} \mathrm{LD}$ indicó la cantidad de pares de ítems con más dependencia local que la sugerida por cada modelo y su magnitud.

\section{Modelización de la ECP con el Modelo de Respuesta Graduada Bifactorial}

Se implementó el MRGB una vez que se comprobó que fue el modelo con mejor ajuste comparativo. Para modelizar la ECP con el MRGB fueron obtenidos, en primer lugar, los parámetros condicionales de inclinación $a_{0}(\mathrm{FG}), a_{1}\left(\mathrm{FE}_{1}\right)$ y $a_{2}$ $\left(\mathrm{FE}_{2}\right)$ y los parámetros de intercepto $c_{1}$ a $c_{5}$, relacionados de forma inversa con la puntuación en el ítem. Los parámetros condicionales de inclinación pueden tener valores distorsionados y resultar complejos de interpretar (Stucky \& Edelen, 2015). Mediante el procedimiento detallado por Stucky y Edelen (2015), se los transformó posteriormente en parámetros marginales de inclinación $a_{0}{ }^{*}, a_{1}{ }^{*}$ y $a_{2}{ }^{*}$ (Stucky, Thissen, \& Edelen, 2013), interpretables de forma similar a los $a$ de los modelos unidimensionales de la TRI. Su significado es reforzado por el cálculo de las cargas factoriales marginales $\lambda_{0}{ }^{*}, \lambda_{1}{ }^{*} \mathrm{y} \lambda_{2}{ }^{*}$. Asimismo, los parámetros $c$ tampoco son de interpretación sencilla. Por esto, se transformaron en 
$b_{k}^{*}$, parámetros marginales de umbral en FG. Los $b_{k}{ }^{*}$ indican el nivel del FG donde la probabilidad de elegir la categoría $k$ o superior es 0.5 , asignando valor nulo a los FE.

El índice de varianza común explicada (Explained Common Variance, ECV) informó el porcentaje de varianza común de las respuestas a los reactivos explicada por el FG (Reise et al., 2010; Berge \& Soèan, 2004). Lo mismo indican los ECV específicos $\left(\mathrm{ECV}_{\mathrm{E}}\right)$ para cada uno de los FE (Toland et al., 2017). También fueron calculados los índices de varianza común explicada por el FG (Item Explained Common Variance, IECV $_{\mathrm{G}}$ ) y los FE (Item Explained Common Variance, $\mathrm{IECV}_{\mathrm{E}}$ ) a nivel del ítem. Estos informan para cada ítem la proporción de varianza común explicada por el FG y por el FE, en este caso según la subescala de pertenencia. Cuanto más alto es el IECV $_{\mathrm{E}}$, más diferentes son los parámetros condicionales y marginales de inclinación entre sí (Toland et al., 2017).

\section{Análisis de confiabilidad}

Se realizó el análisis de confiabilidad global mediante el coeficiente omega, incluyendo sus intervalos de confianza. Se calculó asimismo la Función de Información (FI) para cada subescala por separado aplicando el MRG. La FI permite conocer para qué niveles de Ayuda y Confortar es más precisa la medida.

\section{Programas utilizados}

Para calcular la distancia de Mahalanobis se utilizó SPSS versión 21. El cálculo de los estadísticos descriptivos y el AFE se realizó con el programa FACTOR ${ }^{\circledR}$ versión 10.5 (Ferrando \& Lorenzo-Seva, 2017). El análisis de datos con TRI se implementó con IRTPRO ${ }^{\circledR}$ versión 4.2 (Cai, Thissen, \& Toit, 2011). El cálculo del coeficiente omega y sus intervalos de confianza fue posible gracias al paquete MBESS del software estadístico R (Kelley \& Lai, 2017).

\section{Resultados}

\section{Estadísticos descriptivos y análisis del Funcionamiento Diferencial del Ítem}

Los estadísticos descriptivos para los 15 ítems administrados se pueden observar en la Tabla 1. Los resultados del test de Wald modificado para cada ítem indicaron el no rechazo de la ausencia de DIF.

\section{Comparación del MRGB con dos modelos alternativos}

Los índices de ajuste para los modelos MRG, MRGM y MRGB se exponen en la Tabla 2. El MRGB fue aquel donde los índices -2LL, AIC y BIC y el estadístico $M_{2}$ fueron menores. Además, $M_{2}$ tuvo un valor $p$ asociado no significativo y un RMSEA asociado de .01, lo que supone un ajuste óptimo. Igualmente, ningún ítem desajustó en los tres modelos. Un solo par de ítems mostró dependencia local positiva en el modelo unidimensional MRG, mientras que en los modelos multidimensionales ningún par de ítems mostró dependencia local. Adicionalmente, el valor máximo del índice $\chi^{2}$ LD decrece a menos de la mitad al comparar MRGM con MRG y a un poco más de un cuarto si se compara MRGB con MRG. Es decir, que MRGB modelizó en mayor medida la dependencia local de los ítems que el otro modelo multidimensional, MRGM.

El MRGB es claramente, de los tres, el modelo con mejor ajuste relativo y además parece ser muy compatible con los datos empíricos obtenidos. Es por ello que se le selecciona para la modelización con Teoría de la Respuesta al Ítem Multidimensional (TRIM) de las respuestas a los ítems de la ECP.

\section{Modelización de la ECP con el MRGB}

Los parámetros condicionales de inclinación $a_{0}, a_{1}$ y $a_{2}$ y los parámetros de intercepto $c$ estimados mediante MRGB se observan en la Tabla 3. El subíndice 0 refiere a FG y a los subíndices 1 y 2 a cada uno de los FE. El subíndice 1 se relaciona con 
Tabla 1

Estadísticos descriptivos de los ítems

\begin{tabular}{rrrrr}
\hline Ítem & $M$ & $D E$ & As & $\mathrm{K}$ \\
\hline 1 & 3.53 & 1.03 & .26 & .39 \\
2 & 2.30 & 1.43 & 1.03 & .29 \\
3 & 4.88 & 1.23 & -.86 & -.10 \\
4 & 4.02 & 1.43 & -.15 & -.93 \\
5 & 3.83 & 1.46 & -.03 & -.91 \\
6 & 3.55 & 1.40 & .25 & -.74 \\
7 & 2.83 & 1.34 & .59 & -.20 \\
8 & 3.03 & 1.27 & .47 & -.16 \\
9 & 2.49 & 1.38 & .97 & .34 \\
10 & 4.34 & 1.31 & -.18 & -.99 \\
11 & 3.03 & 1.43 & .46 & -.51 \\
12 & 2.46 & 1.32 & .80 & .05 \\
13 & 4.56 & 1.29 & -.39 & -.97 \\
14 & 5.02 & 1.20 & -.96 & -.17 \\
15 & 4.30 & 1.42 & -.36 & -.89 \\
\hline
\end{tabular}

Nota: $M=$ Media aritmética; $D E$ = Desviación estándar; As = Índice de asimetría; $\mathrm{K}$ = Índice de curtosis.

\section{Tabla 2}

Resultados del ajuste de los modelos MRG, MRGM y MRGB a la ECP

\begin{tabular}{lcccccccccc}
\hline Modelo & -2LL & AIC & BIC & $M_{2}$ & $g l$ & $p$ & RMSEA & \# ít. & \# DL & $\chi^{2}$ LD Máx $^{2}$ \\
\hline MRG & 28694.47 & 28874.47 & 29279.73 & 88.00 & 30 & .0001 & .05 & 15 & 1 & 20.3 \\
MRGM & 28220.18 & 28402.18 & 28811.93 & 77.98 & 29 & .0001 & .05 & 15 & 0 & 9.5 \\
MRGB & 28007.88 & 28217.88 & 28690.68 & 16.80 & 15 & .3327 & .01 & 15 & 0 & 5.3 \\
\hline
\end{tabular}

Nota: MRG = Modelo de Respuesta Graduada (unidimensional); MRGM = Modelo de Respuesta Graduada Multidimensional de dos factores correlacionados; MRGB = Modelo de Respuesta Graduada Bifactorial; ECP = Escala de Conducta Prosocial; -2LL = índice -2 por el logaritmo de la verosimilitud; AIC = Criterio de Información de Akaike; BIC = Criterio de Información Bayesiano; $M_{2}=$ estadístico $M_{2}$ de ajuste global; gl = grados de libertad; RMSEA = error medio cuadrático de aproximación; \# ít. = cantidad de ítems que ajustan al modelo; \# DL = cantidad de pares de ítems con dependencia local detectados; $\chi^{2} \mathrm{LD}_{\text {Máx }}=$ valor máximo del índice $\chi^{2} \mathrm{LD}$.

la subescala Confortar y el subíndice 2 con la subescala Ayuda.

Los parámetros marginales de inclinación $a_{0}{ }^{*}, a_{1}{ }^{*}$ y $a_{2}{ }^{*}$; las cargas factoriales marginales $\lambda_{0}{ }^{*}, \lambda_{1}{ }^{*} \mathrm{y} \lambda_{2}{ }^{*}$ y los parámetros marginales de umbral en FG, $b_{1}^{*}$, $b_{2}^{*}, b_{3}^{*} b_{4}^{*}$ y $b_{5}^{*}$, se encuentran en la Tabla 4 . Asimismo, allí se exponen los índices IECV $_{\mathrm{G}}$ e $\mathrm{IECV}_{\mathrm{E}}$ para cada ítem.
Tanto para la Tabla 3 como para la Tabla 4, se informan los parámetros asociados a FG y al FE en el que está incluido el ítem.

Los ítems con más capacidad de distinguir entre participantes con distintos niveles de FG en orden decreciente según sus valores en los parámetros $a_{0}$ * son 5, 10, 3, 4 y 6. La observación de los parámetros 
$a_{1}^{*}$ indica que los ítems con más capacidad discriminativa entre participantes con distintos niveles de Confortar son el 13 y el 14. Con respecto a Ayuda, los ítems con mayor valor en el parámetro $a_{2}{ }^{*}$ fueron 2, 6, 12 y 11 .

Los ítems cuyas respuestas fueron más determinadas por FG, explicando este factor más del 85\% en su varianza común según el índice $\operatorname{IECV}_{\mathrm{G}} \mathrm{y}$ con elevados valores de $\lambda_{0}{ }^{*}$, fueron 5, 10, 3 y 4 . Existe otro conjunto de ítems con cargas factoriales marginales relativamente similares en FG y en el FE al que pertenecen, lo cual se refleja también en la relación más pareja entre su $\operatorname{IECV}_{\mathrm{G}}$ e $\operatorname{IECV}_{\mathrm{E}}: 1,6,7$, 8, 9, 13, 14 y 15. Esto significa que las respuestas a estos ítems son explicadas tanto por FG como por las subescalas Confortar o Ayuda, según corresponda. En relación con las respuestas a los ítems 2, 11 y 12, las mismas fueron explicadas en muy alta proporción por la subescala Ayuda y en muy baja por FG.

Los parámetros $b_{k}$ * se encuentran en un rango de valores relativamente esperables para los ítems 4,5 , 6, 8, 9 y 10. El resto de los ítems muestran parámetros $b_{k}{ }^{*}$ que exceden sustantivamente el rango -3 a 3. Esto se presentó particularmente en los $b_{k}{ }^{*}$ de categorías extremas. Por otra parte, existen sin duda en la ECP ítems con diferentes niveles de FG requerido para que un participante se ubique en cada uno de $\operatorname{los} b_{k}{ }^{*}$; desde reactivos en los que es suficiente un nivel bajo a medio de FG para alcanzar cualquiera de los $b_{k}{ }^{*}$, como el 3 y el 14, otros ítems donde las distancias entre los $b_{k}{ }^{*}$ son amplias y recorren FG, como el 4 y el 11, hasta ítems exigentes en cuanto al nivel de FG necesario para alcanzar desde $b_{2}{ }^{*}$ en adelante, como el 2, el 9 y el 12.

\section{Tabla 3}

Parámetros estimados mediante el Modelo de Respuesta Graduada Bifactorial para los ítems de la ECP

\begin{tabular}{ccccccccc}
\hline Ítem & $a_{0}($ s.e. $)$ & $a_{1}($ s.e. $)$ & $a_{2}($ s.e. $)$ & $c_{1}($ s.e. $)$ & \multicolumn{1}{c}{$c_{2}($ s.e. $)$} & \multicolumn{1}{c}{$c_{3}($ s.e. $)$} & \multicolumn{1}{c}{$c_{4}($ s.e. $)$} & \multicolumn{1}{c}{$c_{5}($ s.e. $)$} \\
\hline 1 & $.87(.11)$ & & $.69(.10)$ & $4.16(.26)$ & $2.56(.15)$ & $-.18(.09)$ & $-2.08(.13)$ & $-3.53(.20)$ \\
2 & $.99(.17)$ & & $2.57(.25)$ & $.79(.15)$ & $-.92(.15)$ & $-3.12(.24)$ & $-4.39(.31)$ & $-5.33(.37)$ \\
3 & $1.54(.14)$ & $.40(.16)$ & & $5.80(.43)$ & $4.35(.26)$ & $2.31(.15)$ & $.96(.11)$ & $-.44(.11)$ \\
4 & $1.30(.12)$ & $.45(.15)$ & & $4.01(.24)$ & $2.34(.14)$ & $.53(.10)$ & $-.57(.10)$ & $-1.81(.13)$ \\
5 & $1.99(.20)$ & $-.29(.20)$ & & $4.19(.30)$ & $2.48(.20)$ & $.24(.12)$ & $-1.06(.14)$ & $-2.46(.20)$ \\
6 & $1.75(.17)$ & & $1.65(.15)$ & $4.70(.28)$ & $2.29(.17)$ & $-.58(.13)$ & $-1.97(.16)$ & $-3.50(.21)$ \\
7 & $1.13(.13)$ & & $1.43(.13)$ & $2.41(.16)$ & $.42(.11)$ & $-1.77(.14)$ & $-2.87(.17)$ & $-4.24(.24)$ \\
8 & $1.28(.13)$ & & $1.11(.12)$ & $3.03(.18)$ & $.94(.12)$ & $-1.39(.12)$ & $-2.72(.16)$ & $-4.10(.23)$ \\
9 & $1.16(.11)$ & & $.81(.10)$ & $1.33(.12)$ & $-.46(.10)$ & $-2.06(.13)$ & $-2.81(.16)$ & $-3.67(.21)$ \\
10 & $1.83(.15)$ & $.40(.17)$ & & $5.79(.41)$ & $4.19(.26)$ & $1.12(.13)$ & $-.23(.12)$ & $-1.56(.14)$ \\
11 & $.81(.13)$ & & $1.57(.13)$ & $2.46(.16)$ & $.71(.12)$ & $-1.30(.13)$ & $-2.42(.16)$ & $-3.64(.21)$ \\
12 & $.93(.13)$ & & $1.65(.14)$ & $1.41(.13)$ & $-.37(.12)$ & $-2.32(.16)$ & $-3.40(.20)$ & $-4.97(.20)$ \\
13 & $.92(.14)$ & $1.34(.21)$ & & $6.18(.54)$ & $4.12(.30)$ & $1.47(.15)$ & $.26(.11)$ & $-1.04(.13)$ \\
14 & $1.61(.21)$ & $1.80(.31)$ & & $8.19(.85)$ & $5.92(.52)$ & $3.04(.28)$ & $1.56(.18)$ & $.02(.14)$ \\
15 & $.68(.10)$ & $.74(.13)$ & & $3.97(.25)$ & $2.58(.15)$ & $.84(.10)$ & $-.02(.09)$ & $-1.16(.10)$ \\
\hline
\end{tabular}

Nota: $a_{0}=$ Parámetro condicional de inclinación en el factor general; $a_{1}=$ Parámetro condicional de inclinación en el factor Confortar; $a_{2}=$ Parámetro condicional de inclinación en el factor Ayuda; $c_{1} \ldots c_{5}=$ Parámetros de intercepto; s.e. = Error estándar. 


\section{Tabla 4}

Parámetros marginales calculados a partir del Modelo de Respuesta Graduada Bifactorial para los ítems de la ECP

\begin{tabular}{rrrrrrrrrrrrrr}
\hline Ítem & $a_{0}{ }^{*}$ & $a_{1}{ }^{*}$ & $a_{2}{ }^{*}$ & $\lambda_{0}{ }^{*}$ & $\lambda_{1}{ }^{*}$ & $\lambda_{2}{ }^{*}$ & $b_{1}{ }^{*}$ & $b_{2}{ }^{*}$ & $b_{3}{ }^{*}$ & $b_{4}{ }^{*}$ & $b_{5}{ }^{*}$ & IECV $_{\mathrm{G}}$ & $\mathrm{IECV}_{\mathrm{E}}$ \\
\hline 1 & .81 & & .64 & .43 & & .35 & -4.78 & -2.94 & .21 & 2.40 & 4.06 & .60 & .40 \\
2 & .55 & & 1.42 & .31 & & .64 & -.80 & .93 & 3.15 & 4.43 & 5.39 & .19 & .81 \\
3 & 1.50 & .39 & & .66 & .22 & & -3.76 & -2.82 & -1.50 & -.62 & -.29 & .90 & .10 \\
4 & 1.26 & .44 & & .59 & .25 & & -3.08 & -1.80 & -.41 & .44 & 1.39 & .85 & .15 \\
5 & 1.96 & -.29 & & .76 & -.17 & & -2.10 & -1.25 & .12 & .53 & 1.24 & .95 & .05 \\
6 & 1.26 & & 1.18 & .59 & & .57 & -2.69 & -1.31 & .33 & 1.13 & 2.00 & .52 & .48 \\
7 & .86 & & 1.09 & .45 & & .54 & -2.13 & -.37 & 1.57 & 2.54 & 3.75 & .41 & .59 \\
8 & 1.07 & & .93 & .53 & & .48 & -2.37 & -.73 & 1.09 & 2.13 & 3.20 & .55 & .45 \\
9 & 1.05 & & .73 & .52 & & .40 & -1.15 & .40 & 1.78 & 2.42 & 3.16 & .64 & .36 \\
10 & 1.78 & .39 & & .72 & .22 & & -3.16 & -2.29 & -.61 & .13 & .85 & .91 & .09 \\
11 & .60 & & 1.15 & .33 & & .56 & -3.03 & -.88 & .60 & 2.99 & 4.49 & .26 & .74 \\
12 & .67 & & 1.18 & .37 & & .57 & -1.52 & .40 & 2.49 & 3.66 & 5.34 & .29 & .71 \\
13 & .72 & 1.05 & & .39 & .53 & & -6.71 & -4.48 & -1.60 & -.28 & 1.13 & .36 & .64 \\
14 & 1.11 & 1.24 & & .55 & .59 & & -5.09 & -3.68 & -1.89 & -.97 & -.01 & .46 & .54 \\
15 & .62 & .68 & & .34 & .37 & & -5.84 & -3.79 & -1.23 & .03 & 1.71 & .46 & .54 \\
\hline
\end{tabular}

Nota: $a_{0}^{*}=$ Parámetro marginal de inclinación en el factor general; $a_{1}^{*}=$ Parámetro marginal de inclinación en el factor Confortar; $a_{2}^{*}=$ Parámetro marginal de inclinación en el factor Ayuda; $\lambda_{0}{ }^{*}, \lambda_{1}{ }^{*}$ y $\lambda_{2}{ }^{*}=$ Cargas factoriales marginales; $b_{1}{ }^{*} \ldots b_{5}^{*}=$ Parámetros marginales de umbral en el factor general; $\mathrm{IECV}_{\mathrm{G}}=$ Índice de varianza común explicada por el factor general a nivel del ítem; IECV $\mathrm{E}_{\mathrm{E}}=$ Índice de varianza común explicada por el factor específico a nivel del ítem.

Por último, el resultado del índice ECV fue de $60.30 \%$, es decir que FG explicó un $60.30 \%$ de la varianza común de las respuestas a los ítems. El ECV específico para la subescala Ayuda fue de 22.88\%, mientras que el ECV específico para la subescala Confortar resultó en $4.52 \%$. Si se suma la varianza común explicada por FG y los dos FE, se halla que dieron cuenta de un total de $87.70 \%$ de la varianza común de las respuestas a la ECP.

\section{Análisis de confiabilidad}

El valor del coeficiente omega para la subescala Confortar fue de .78 con un IC al 95\% de [.750,
.804]. Para la subescala Ayuda el coeficiente omega fue de .85 con un IC al 95\% de [.835, .872].

\section{Función de Información de la subescala Confortar}

La Figura 1 muestra que la FI del test resultó ligeramente decreciente y alcanzó su valor máximo de 5.60 en $\theta=-.30$. Como es de esperar, dada su relación inversa, el error de medida alcanzó en este punto su valor mínimo de .42. Esta función muestra que el test proporciona estimaciones de Confortar más precisas en los niveles de rasgo zeta de -3 a 1.80, mientras que el error en la medición crece hacia los niveles del extremo superior del rasgo Confortar. 


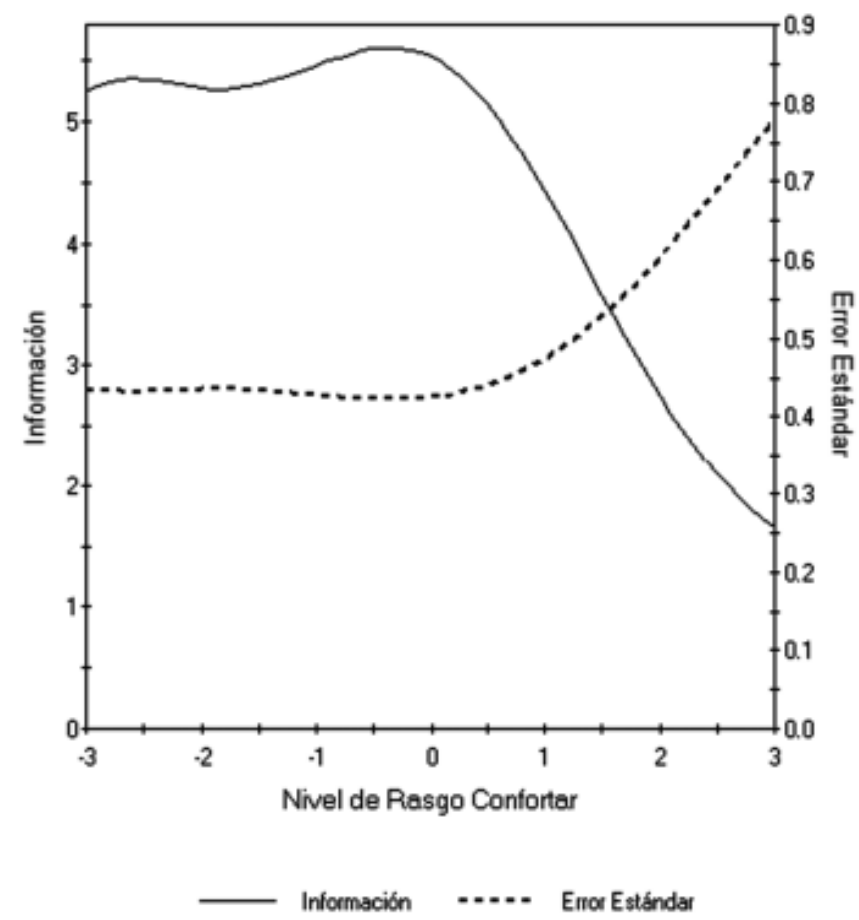

Figura 1. Función de Información del test y error estándar en la subescala Confortar.

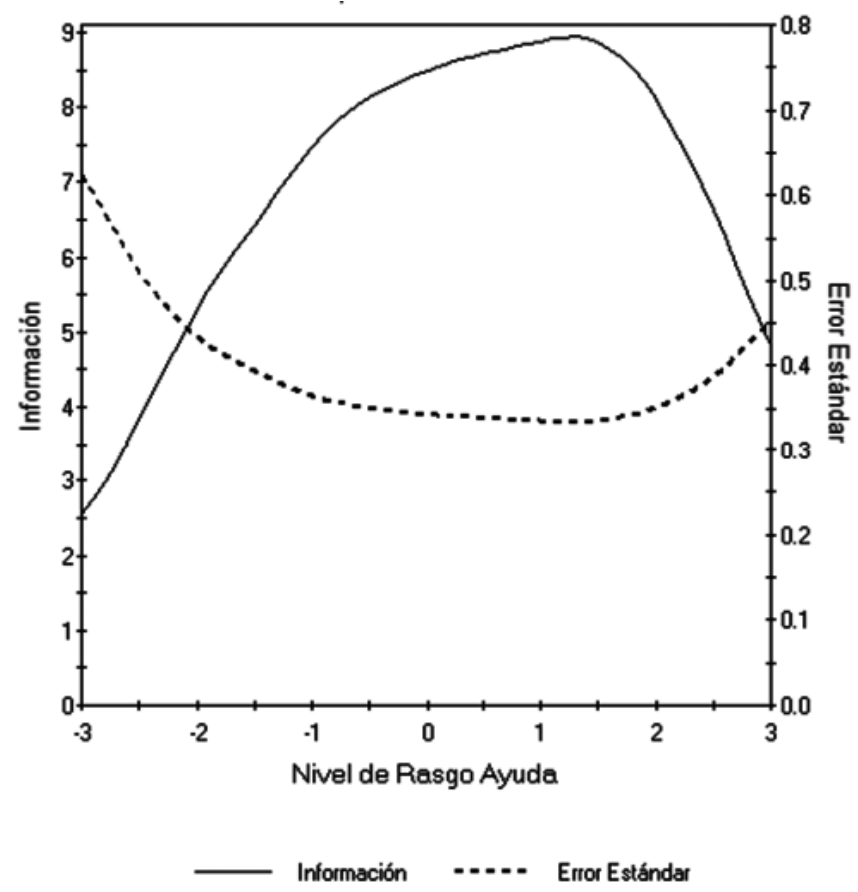

Figura 2. Función de Información del test y error estándar en la subescala Ayuda. 


\section{Discusión}

Los resultados del presente estudio sostuvieron la hipótesis de que el modelo bifactorial fue el que mejor ajustó a los ítems de la ECP. La mayoría de las investigaciones, hasta el momento, se habían concentrado ya sea en la prosocialidad como tendencia general de la personalidad (e.g., Caprara et al., 2012, Ferguson, Zhao, O’Carroll, \& Smillie, 2018) o en las conductas prosociales específicas, considerando a la conducta prosocial como un constructo dividido en subtipos (e.g., Dunfield, 2014; Eisenberg, \& Spinrad, 2014). Los estudios centrados en las conductas prosociales específicas utilizaron un enfoque clásico que no consideró la existencia de un factor general subyacente. En la presente investigación, aunque el modelo de dos factores correlacionados tuvo mejor ajuste que el modelo unidimensional, fue el modelo bifactorial el que se adaptó óptimamente a los datos. Esta mejora significativa en el ajuste fue lograda gracias a que el modelo bifactorial tomó en cuenta la existencia del factor general. Es decir, que el presente estudio provee apoyo empírico a la hipótesis de la existencia de la prosocialidad, la tendencia general de la personalidad a realizar acciones prosociales (Caprara et al., 2012). Por supuesto, se trata de una contribución adicional a muchas otras que se pueden realizar en este sentido.

Los ítems de la ECP fueron influenciados primariamente por la prosocialidad. En segundo lugar, la subescala Ayuda explicó una proporción importante de la varianza común, destacándose su importancia como conducta prosocial específica. En cambio, la subescala Confortar, considerada como factor específico, explicó una parte pequeña de dicha varianza. La implementación del MRGB también indicó que cuatro de los ítems de la ECP fueron influenciados casi exclusivamente por la prosocialidad, sin cargar significativamente en ningún factor específico. Tres de esos cuatro ítems refieren a sentimientos o comportamientos empáticos que se habían incluido en la subescala Confortar. Estos hallazgos podrían estar sugiriendo que la capacidad empática autopercibida es un componente esencial de la prosocialidad.

En este sentido, la empatía había sido planteada clásicamente como un fuerte predictor de la tendencia a realizar acciones prosociales basado esto, en general, en elevadas correlaciones entre instrumentos que miden ambos constructos (e.g., Mestre, Samper, \& Frías, 2002). Caprara et al. (2005), sostuvieron otra postura: la empatía es parte de la prosocialidad en la etapa adulta. Para ellos, en la adolescencia y adultez las tendencias empáticas simplemente no se asocian con la prosocialidad sino que son un componente integral de la misma. Realizar acciones prosociales supone la capacidad de sentir las necesidades y estados de ánimo ajenos. Esta contraposición de definiciones fue destacada previamente por Auné, Abal y Attorresi (2016).

Los resultados del presente estudio proveen una posible explicación para las diferentes estructuras alternativas que se han hallado para la conducta prosocial. Asimismo, contribuyen en clarificar la existencia de la prosocialidad y su naturaleza. Resultados coherentes con estos hallazgos han sido encontrados por Knafo-Noam, Uzefovsky, Israel, Davidov y Zahn-Waxler (2015) quienes estudiaron el comportamiento prosocial de gemelos y mellizos israelíes de edad promedio 90 meses mediante un análisis factorial confirmatorio clásico con el modelo common-factor-common-pathways multivariate model. Knafo-Noam et al. concluyeron que un único factor subyacente explicaba fuertemente la varianza común y lo relacionaron con la prosocialidad o personalidad prosocial. Este factor subyacente tuvo un efecto muy importante de la carga genética, aunque también fue explicado en parte por efectos medioambientales.

Se concluye que la estructura de la conducta prosocial parece repetirse en distintas culturas y ciclos vitales, aunque sería necesario realizar investigaciones adicionales con una diversidad en cuanto a las poblaciones de referencia y en los instrumentos de 
medición utilizados. Esta es una meta para futuros estudios.

Por último, el hecho de que los parámetros de umbral de varios ítems hayan excedido el rango -3 a 3 puede estar indicando que sea deseable colapsar las categorías de respuesta Nunca y Casi Nunca, así como Casi Siempre y Siempre. Es decir que un diseño de cuatro opciones de respuesta debería ser testeado para la ECP. Esta cantidad de opciones ha sido considerada una cantidad óptima de categorías en anteriores estudios (e.g., Abal, Auné, \& Attorresi, 2018; Lozano, García-Cueto, \& Muñiz, 2008).

En cuanto a las limitaciones del presente estudio se destaca lo siguiente: la muestra no es representativa de la población argentina, sería útil relacionar estos resultados con estudios de análisis factorial confirmatorio tradicional, y sería importante relacionar los puntajes en la ECP con medidas de deseabilidad social.

\section{Conflicto de intereses}

No existe ningún conflicto de interés.

\section{Responsabilidad ética}

Se aclaró que la participación podía cesar en cualquier momento y que se resguardaría la anonimidad de las respuestas. Los autores declaran que han seguido los protocolos de su centro de afiliación sobre el cuidado de los participantes de este estudio. Los datos de los participantes son anónimos.

\section{Agradecimientos}

Esta investigación se ha realizado dentro del proyecto UBACyT 2018 con código 20020170 100200BA, subvencionado por la Facultad de Psicología de la Universidad de Buenos Aires, el proyecto UBACyT 2018 con código 2002017020 0001BA subvencionado también por la Facultad de Psicología de la Universidad de Buenos Aires y el proyecto PICT-2017-3226 subvencionado por la
Agencia Nacional de Promoción Científica y Tecnológica.

\section{Contribución de autoría}

SEA: concepción y diseño del estudio, recolección de los datos, análisis estadístico e interpretación de los datos.

FJPA: concepción y diseño del estudio, interpretación de los datos y revisión final del manuscrito.

HFA: concepción y diseño del estudio, interpretación de los datos y revisión final del manuscrito.

\section{Referencias}

Abal, F. J. P., Auné, S. E., \& Attorresi, H. F. (2018). Variación de la escala Likert en el test de Utilidad de la Matemática. Interacciones, 4(3), 177-189. doi: 10. 24016/2018.v4n3.134

American Psychological Association (2010). Ethical Principles for Psychologists and Code of Conduct. Washington, D.C.: APA.

Auné, S. E., Abal, F. J. P., \& Attorresi, H. F. (2016). Antagonismos entre concepciones de empatía y su relación con la conducta prosocial. Revista de Psicología, 17(2), 137-149. doi: 10.18050/revp si.v17n2a7.2015

Auné, S. E., \& Attorresi, H. F. (2017). Dimensionalidad de un Test de Conducta Prosocial. Revista Evaluar, 17(1), 29-37. Recuperado de https://revistas.unc.edu. ar/index.php/revaluar/article/view/17072

Auné, S. E., Blum, G. D., Abal, F. J. P., Lozzia, G. S., \& Attorresi, H. F. (2014). La conducta prosocial: Estado actual de la investigación. Perspectivas en Psicología, 11(2), 21-33.

Ayala, R. J. de (2009). The theory and practice of item response theory. New York: Guilford Press.

Benjamini, Y., \& Hochberg, Y. (1995). Controlling the False Discovery Rate: A Practical and Powerful Approach to Multiple Testing. Journal of the Royal Statistical Society, Series B, 57(1), 289-300. doi: 10.1111/j.2517-6161.1995.tb02031.x 
Berge, J. M. ten, \& Soèan G. (2004). The greatest lower bound to the reliability of a test and the hypothesis of unidimensionality. Psychometrika, 69(4), 613625. doi: $10.1007 / \mathrm{bf02289858}$

Bjorner, J. B., Smith, K. J., Edelen, M. O., Stone, C., Thissen, D., \& Sun, X. (2007). IRTFIT: A macro for item fit and local dependence tests under IRT models. Lincoln, RI: QualityMetric Incorporated.

Bock, R. D., \& Gibbons, R. (2010). Factor analysis of categorical item responses. In M. L. Nering \& R. Ostini (Eds.), Handbook of polytomous item response theory models (pp. 155-184). New York: Routledge.

Cai, L. (2012). flexMIRT: Flexible multilevel item factor analysis and test scoring [Computer software]. Seattle, WA: Vector Psychometric Group, LLC.

Cai, L., Thissen, D., \& Toit, S. du (2011). IRTPRO user's guide. Lincolnwood, IL: Scientiûc Software International.

Cai, Y. (2015). The value of using test response data for content validity: An application of the bifactor-MIRT to a nursing knowledge test. Nurse Education Today, 35(12), 1181-1185. doi: 10.1016/j.nedt.2015.05.014

Caprara, G. V., Alessandri, G., \& Eisenerg, N. (2012). Prosociality: the contribution of traits, values, and self-efficacy beliefs. Journal of Personality and Social Psychology, 102(6), 1289-1303. doi: 10.103 7/a0025626

Caprara, G. V., \& Pastorelli, C. (1993). Early emotional instability, prosocial behavior, and aggression: some methodological aspects. European Journal of Personality, 7(1), 19-36. doi: 10.1002/per.241007 0103

Caprara, G. V., Steca, P., Zelli, A., \& Capanna, C. (2005). A new scale for measuring adults' prosocialness. European Journal of Psychological Assessment, 21(2), 77-89. doi: 10.1027/1015-5759.21.2.77

Carlo, G., \& Randall, B. A. (2002). The Development of a Measure of Prosocial Behaviors for Late Adolescents. Journal of Youth and Adolescence, 31(1), 31-44. doi: 10.1023/A:1014033032440
Carrasco, C., \& Trianes, M. V. (2010). Clima social, prosocialidad y violencia como predictores de inadaptación escolar en primaria. European Journal of Education and Psychology, 3(2), 229-242. doi: 10.30552/ejep.v3i2.54

Chen, W., \& Thissen, D. (1997). Local dependence indixes for item pairs using item response theory. Journal of Educational and Behavioral Statistics, 22, 265-289. doi: 10.3102/10769986022003265

Cuadrado, E., Tabernero, C., García, R., Luque, B., \& Seibert, J. (2017). The Role of Prosocialness and Trust in the Consumption of Water as a Limited Resource. Frontiers in Psychology, 8, 694. doi: 10.3 389/fpsyg.2017.00694

Dunfield, K. A. (2014). A construct divided: prosocial behavior as helping, sharing, and comforting subtypes. Frontiers in Psychology, 5, 958. doi: 10.3389/fpsy g.2014.00958

Eisenberg, N., Eggum, N. D., \& Giunta, L. di (2010). Empathy-Related Responding: Associations with Prosocial Behavior, Aggression, and Intergroup Relations. Social Issues and Policy Review, 4(1), 143-180. doi: 10.1111/j.1751-2409.2010.01020.x

Eisenberg, N., \& Spinrad, T. L. (2014). Multidimensionality of prosocial behavior. Rethinking the conceptualization and development of prosocial behavior. In L. M. PadillaWalker \& G. Carlo (Eds.), Prosocial development: A multidimensional approach (pp. 17-39). Oxford: Oxford University Press. doi: 10.1093/acprof:oso/ 9780199964772.003 .0002

Ferguson, E., Zhao, K., O’Carroll, R. E., \& Smillie, L. D. (2018). Costless and Costly Prosociality: Correspondence Among Personality Traits, Economic Preferences, and Real-World Prosociality. Social Psychological and Personality Science, 10(4), 461471. doi: 10.1177/1948550618765071

Ferrando, P. J., \& Lorenzo-Seva, U. (2017). Program FACTOR at 10: Origins, development and future directions. Psicothema, 29(2), 236-240. doi: 10.7334/ psicothema2016.304 
Gatner, D. T., Douglas, K. S., \& Hart, S. D. (2016). Examining the incremental and interactive effects of boldness with meanness and disinhibition within the triarchic model of psychopathy. Personality Disorders: Theory, Research, and Treatment, 7(3), 259-268. doi: 10.1037/per0000182

Gibbons, R. D., Bock, R. D., Hedeker, D., Weiss, D. J., Segawa, E., Bhaumik, D. K., ... Stover, A. (2007). FullInformation Item Bifactor Analysis of Graded Response Data. Applied Psychological Measurement, 31(1), 4-19. doi: 10.1177/0146621606289485

Gibbons, R. D., \& Hedeker, D. R. (1992). Fullinformation bi-factor analysis. Psychometrika, 57(3), 423-436. doi: 10.1007/bf02295430

González, M. D. (2000). Conducta prosocial: Evaluación e Intervención. Madrid, España: Morata.

Hay, D. F., \& Cook, K. V. (2007). The transformation of prosocial behavior from infancy to childhood. In C. A. Brownell \& C. B. Kopp (Eds.), Socioemotional development in the toddler years: Transitions and transformations (pp. 100-131). New York: The Guilford Press.

Hu, L., \& Bentler, P. M. (1999). Cutoff criteria for fit indexes in covariance structure analysis: Conventional criteria versus new alternatives. Structural Equation Modeling: A Multidisciplinary Journal, 6, 1-55. doi: 10.1080/10705519909540118

Inderbitzen, H. M., \& Foster, S. L. (1992). The Teenage Inventory of Social Skills: Development, reliability, and validity. Psychological Assessment, 4(4), 451459. doi: 10.1037/1040-3590.4.4.451

Kelley, K., \& Lai, K. (2017). The MBESS R Package version 4.2.0. Recuperado de https://cran.r-project. org/web/packages/MBESS/MBESS.pdf

Knafo-Noam, A., Uzefovsky, F., Israel, S., Davidov, M., \& Zahn-Waxler, C. (2015). The prosocial personality and its facets: genetic and environmental architecture of mother-reported behavior of 7-year-old twins. Frontiers in Psychology, 6, 112. doi: 10.3389/fpsy g.2015.00112
Ladd, G. W., \& Profilet, S. M. (1996). The Child Behavior Scale: A teacher-report measure of young children's aggressive, withdrawn, and prosocial behaviors. Developmental Psychology, 32(6), 1008-1024. doi: 10.1037/0012-1649.32.6.1008

Langer, M. (2008). A reexamination of Lord's Wald test for differential item functioning using item response theory and modern error estimation (Unpublished doctoral dissertation). Recuperada de https://pdfs. semanticscholar.org/204e/a4e24df2284f4c6833 b73ec18a01964fe2ca.pdf

Lozano, L. M., García-Cueto, E., \& Muñiz, J. (2008). Effect of the Number of Response Categories on the Reliability and Validity of Rating Scales. Methodology, 4(2), 73-79. doi: 10.1027/1614-2241.4.2.73

Maydeu-Olivares, A., \& Joe, H. (2005). Limited and full information estimation and testing in $2^{n}$ Contingency Tables: A Unified Framework. Journal of the American Statistical Association, 100, 1009-1020. doi: 10.1198/016214504000002069

Maydeu-Olivares, A., \& Joe, H. (2006). Limited Information Goodness-of-fit Testing in Multidimensional Contingency Tables. Psychometrika, 71, 713-732. doi: 10.1007/s11336-005-1295-9

Mestre, M. V., Samper, P., \& Frías, M. D. (2002). Procesos cognitivos y emocionales predictores de la conducta prosocial y agresiva: La empatía como factor modulador. Psicothema, 14(2), 227-232.

Muraki, E., \& Carlson, J. E. (1995). Full-Information Factor Analysis for Polytomous Item Responses. Applied Psychological Measurement, 19(1), 73-90. doi: 10.1177/014662169501900109

Orlando, M., \& Thissen, D. (2000). Likelihood-Based Item Fit Indices for Dichotomous Item Response Theory Models. Applied Psychological Measurement, 24(1), 50-64. doi: 10.1177/01466216000241003

Orlando, M., \& Thissen, D. (2003). Further Investigation of the Performance of $S-\chi^{2}$ : An Item Fit Index for Use With Dichotomous Item Response Theory Models. Applied Psychological Measurement, 27(4), 289298. doi: 10.1177/0146621603027004004 
Ouyang, X., Xin, T., \& Chen, F. (2016). Construct Validity of the Children's Coping Strategies Scale (CCSS) A Bifactor Model Approach. Psychological Reports, 118(1), 199-218. doi: 10.1177/0033294116628362

Penner, L., Fritzsche, B., Craiger, J., \& Freifeld, T. (1995). Measuring the prosocial personality. In J. Butcher, \& C. Spielberger (Eds.), Advances in Personality Assessment (Vol. 10). Hillsdale, NJ: Erlbaum.

Reise, S. P., Moore, T. M., \& Haviland, M. G. (2010). Bifactor Models and Rotations: Exploring the Extent to Which Multidimensional Data Yield Univocal Scale Scores. Journal of Personality Assessment, 92(6), 544-559. doi: 10.1080/00223891.2010.496477

Roche, R. (1998). El uso educativo de la televisión como optimizadora de la prosocialidad. Psychosocial Intervention, 7(3), 363-377. Recuperado de http:// www.copmadrid.org/webcopm/publicaciones/social/ 43319.pdf

Samejima, F. (1968). Estimation of latent ability using a response pattern of graded scores. Psychometrika monograph supplement, 17(4), 2. doi: 10.1002/j.23 33-8504.1968.tb00153.x

Strakatý, S. (2016). Relationship between Traumatic Experience and Prosocial Behavior (Tesis de bachillerato). State University of New York, Empire State College.

Stucky, B. D., \& Edelen, M. O. (2015). Using hierarchical IRT models to create unidimensional measures from multidimensional data. In S. P. Reise \& D. A. Revicki (Eds.), Handbook of item response theory modeling: Applications to typical performance assessment (pp. 183-206). New York: Routledge.

Stucky, B. D., Thissen, D., \& Edelen, M. O. (2013). Using Logistic Approximations of Marginal Trace Lines to Develop Short Assessments. Applied Psychological Measurement, 37(1), 41-57. doi: 10.1177/0146621 612462759

Tabachnick, B. G., \& Fidell, L. S. (2001). Using multivariate statistics. Needham Heights, MA: Ally and Bacon.

Toland, M. D., Sulis, I., Giambona, F., Porcu, M., \& Campbell, J. M. (2017). Introduction to bifactor polytomous item response theory analysis. Journal of School Psychology, 60, 41-63. doi: 10.1016/j.jsp. 2016.11.001

Warneken, F., \& Tomasello, M. (2009). The roots of human altruism. British Journal of Psychology, 100(3), 455-471. doi: 10.1348/000712608X379061

Sofía Esmeralda Auné

Instituto de Investigaciones de la Facultad de Psicología, Universidad de Buenos Aires, Argentina; Consejo Nacional de Investigaciones Científicas y Técnicas (CONICET), Argentina

ORCID: 0000-0002-0620-0199

Autor corresponsal: sofiaaune177@hotmail.com

Facundo Juan Pablo Abal

Instituto de Investigaciones de la Facultad de Psicología, Universidad de Buenos Aires, Buenos Aires, Argentina; Consejo Nacional de Investigaciones Científicas y Técnicas (CONICET), Argentina

ORCID: 0000-0001-7023-5380

afjp79@hotmail.com

Horacio Félix Attorresi

Instituto de Investigaciones de la Facultad de Psicología, Universidad de Buenos Aires, Argentina

ORCID: 0000-0002-3027-1069 